\title{
miR-137 acts as a tumor suppressor in papillary thyroid carcinoma by targeting CXCL12
}

\author{
SU DONG ${ }^{1}$, MEISHAN JIN ${ }^{2}, \mathrm{YE} \mathrm{LI}^{3}$, PEIYOU REN ${ }^{4}$ and JIA LIU ${ }^{4}$ \\ Departments of ${ }^{1}$ Anesthesia, ${ }^{2}$ Pathology, ${ }^{3}$ Radiology and ${ }^{4}$ Thyroid Surgery, \\ The First Hospital of Jilin University, Chaoyang, Changchun, Jilin 130021, P.R. China
}

Received November 11, 2015; Accepted December 18, 2015

DOI: $10.3892 /$ or.2016.4604

\begin{abstract}
Accumulating evidence has shown that aberrantly expressed microRNAs (miRs) are extensively involved in tumorigenesis. microRNA-137 (miR-137) has been reported as a tumor suppressor in various types of cancer. However, the biological function and underlying molecular mechanism of miR-137 in papillary thyroid carcinoma (PTC) remain largely unknown. Therefore, the present study aimed to investigate the expression pattern of miR-137 and its functional significance in PTC. Quantitative RT-PCR (qRT-PCR) assay showed that miR-137 expression was significantly downregulated in human PTC tissues, and its expression was significantly negatively correlated with tumor-node-metastasis (TNM) stage and lymph node metastasis. Functional assays showed that forced expression of miR-137 in PTC cells significantly inhibited proliferation, colony formation, migration and invasion in vitro. Importantly, on the basis of bioinformatic analysis and luciferase reporter assay, we found that miR-137 directly targeted the 3'-untranslated region (3'-UTR) of C-X-C motif chemokine 12 (also known as SDF-1) (CXCL12). qRT-PCR and western blot analysis further verified the results and demonstrated that $\mathrm{miR}-137$ could downregulate CXCL12 expression in PTC cells. We also confirmed that CXCL12 expression was increased in PTC tissues and was inversely correlated with miR-137. In addition, our results also showed that downregulation of CXCL12 mimicked the effects of miR-137 overexpression, and upregulation of CXCL12 partially reversed the inhibitory effects of miR-137 in PTC cells. These results showed that miR-137 may function as a tumor suppressor in PTC by targeting CXCL12, suggesting that miR-137 may act as a potential target for PTC treatment.
\end{abstract}

Correspondence to: Dr Jia Liu, Department of Thyroid Surgery, The First Hospital of Jilin University, 71 Xinmin Street, Chaoyang, Changchun, Jilin 130021, P.R. China

E-mail: liujia1538@sina.com

Key words: papillary thyroid carcinoma, miR-137, CXCL12, proliferation

\section{Introduction}

Thyroidcancer (TC) is the most common endocrine malignancy, and papillary thyroid carcinoma (PTC) is the most prevalent type of tumor among thyroid malignancies, accounting for $\sim 80 \%$ of all TC cases $(1,2)$. Currently, although the majority of PTC cases have excellent prognosis and therapeutic response with a combination of radioiodine and levothyroxine after complete thyroidectomy (3), $10 \%$ of cases present recurrence in local/regional and distant sites within 10 years leading to death (4). A larger number of studies have been conducted on the pathogenesis of PTC, yet, the underlying mechanisms of the tumorigenesis and metastasis of PTC remain largely unclear. Consequently, a better understanding of the mechanisms involved in PTC tumorigenesis and metastasis is very important for its prevention, diagnosis and treatment.

microRNAs (miRNAs) are small (19-25 nucleotides), single-stranded, non-coding RNA molecules that regulate gene expression by interacting with the 3'-untranslated region (3'-UTR) of messenger RNAs (mRNAs), leading to reduce the stability and/or translation efficiency of target mRNAs in a sequence-specific manner (5-7). Increasing evidence has shown that miRNAs are involved in various biological processes, including cell proliferation, apoptosis, cell cycle and invasion $(8,9)$. Numerous known miRNAs have been reported to play crucial roles involved in tumorigenesis and/ or metastasis by directly targeting molecular targets $(10,11)$. A recent study suggested that differentially expressed miRNAs in PTC can be used as potential diagnostic and therapeutic targets (12). Therefore, there is a need to characterize novel miRNAs involved in PTC tumorigenesis and metastasis, which can provide a new insight into the diagnosis, prognosis and therapy for this disease.

microRNA-137 (miR-137) functions as a tumor suppressor in many types of human cancers, including gastric (13), colorectal (14), non-small cell lung (15) and ovarian cancer (16), neuroblastoma (17) and breast cancer (18). However, the clinical significance, and its role and underlying molecular mechanism in PTC remain unclear. Therefore, in the present study, we analyzed the association of miR-137 expression with clinicopathologic features in patients with PTC. In addition, we also investigated the potential role of miR-137 and the underlying mechanism in PTC using a series of molecular and cellular experiments. 


\section{Materials and methods}

PTC tissue samples. Paired adjacent normal and PTC tumor tissue samples were obtained from 30 PTC patients who underwent surgical resection at the Department of Thyroid Surgery, First Hospital of Jilin University (Changchun, China). All samples were immediately frozen in liquid nitrogen and stored at $-80^{\circ} \mathrm{C}$ until use. The characteristics of the patients are described in Table I. All patients provided written informed consent for the use of their tissues. The present study was approved by the Ethics Committee of Jilin University.

Cell lines and cell culture. The human PTC cell line, K1, was obtained from the Type Culture Collection of the Chinese Academy of Sciences (Shanghai, China), and was cultured in RPMI-1640 medium (Invitrogen, Carlsbad, CA, USA) supplemented with $10 \%$ fetal bovine serum (FBS; Gibco-BRL) and $1 \%$ streptomycin-penicillin (Sigma, St. Louis, MO, USA) at $37^{\circ} \mathrm{C}$ in a humidified atmosphere containing $5 \% \mathrm{CO}_{2}$.

RNA extraction and quantitative RT-PCR ( $q R T-P C R)$. Total RNAs including miRNAs from tissues and cells were isolated using the miRNeasy Mini kit (Qiagen, Dusseldorf, Germany) according to the manufacturer's protocol. For detection of miR-137, first-strand cDNA was synthesized using miScript reverse transcription kit (Qiagen). The specific primers of miR-137 and U6 were used as previously described (19). U6 small nuclear RNA was used as an internal control. Amplification procedures were performed on an ABI 7900 real-time PCR system (Applied Biosystems, Carlsbad, CA, USA) using TaqMan miRNA assay kits (Applied Biosystems, Foster City, CA, USA). To quantify CXCL12, total RNA was reversely transcribed into cDNA using the PrimeScript RT reagent kit (Takara, Dalian, China). Amplification procedures were performed on an ABI 7900 real-time PCR system using real-time PCR mixture reagent (Takara). The specific primers of CXCL12 and GAPDH were used as previously described (20). GAPDH was used as an internal control. The relative gene expression was analyzed using the $2^{-\Delta \Delta C t}$ method.

Cell transfection. miR-137 mimics (miR-137) and the corresponding miRNA negative control (miR-NC) were purchased from Qiagen (Frederick, MD, USA). The small interfering RNAs (siRNAs) targeting human CXCL12 (si-CXCL12) and the corresponding negative control (si-NC) and the overexpression CXCL12 plasmid were purchased from GenePharma (Shanghai, China). For transfection, K1 cells were seeded into 6-well plates for $24 \mathrm{~h}$ and then transiently transfected with these molecular products using Lipofectamine 2000 (Invitrogen) according to the manufacturer's instructions.

MTT assay. Transfected cells $\left(5 \times 10^{3}\right.$ cells/well) were seeded into 96-well plates and cultured in Dulbecco's modified Eagle's medium (DMEM) including 10\% FBS. At the indicated times (24, 48 and $72 \mathrm{~h}$ ), MTT solution ( $5 \mathrm{mg} / \mathrm{ml}$; Sigma) was added to each well with fresh medium for $4 \mathrm{~h}$. Afterwards, dimethyl sulfoxide (DMSO) $(150 \mu \mathrm{l} /$ well) was added to dissolve the formazan product for $\sim 15 \mathrm{~min}$. The absorption at $570 \mathrm{~nm}$ was measured under a multi-well spectrophotometer (Bio-Tek Instruments, Winooski, VT, USA).
Colony formation assay. Transfected cells were seeded into a 6-well plate in RPMI-1640 medium, supplemented with 10\% FBS and $0.3 \%$ noble agar at 200 cells/well to form natural colonies. After two weeks, the cells were washed with phosphate-buffered saline (PBS), fixed with 4\% paraformaldehyde for $20 \mathrm{~min}$, and stained with Giemsa (Sigma). The total number of colonies was counted under a light microscope (Olympus, Tokyo, Japan).

Wound healing assay. Approximately $1 \times 10^{5}$ transfected cells were seeded into each well of a 6-well plate. When cell confluence reached $\sim 90-100 \%$, wounds were created in the confluent cells using a $200-\mu 1$ pipette tip. After wounding, the debris was removed by washing the cells with medium, and then medium was added and cultured for $24 \mathrm{~h}$. Wound healing was observed and photographed at different time points $(0,24$ and $48 \mathrm{~h}$ ) within the scrape line using a phase contrast microscope (Olympus). The results were quantified using ImageJ software.

Cell invasion assay. A Transwell invasion assay was performed with $8.0-\mathrm{mm}$ pore filters according to the manufacturer's instructions (BD Biosciences, San Jose, CA, USA). Briefly, the $2 \times 10^{4}$ transfected cells were added to the upper Transwell chambers coated with Matrigel (BD Biosciences, Bedford, MA, USA) in serum-free medium. RPMI-1640 medium containing $10 \%$ FBS was added into the lower chamber as the chemoattractant. Afterwards, the cells were incubated for $48 \mathrm{~h}$ at $37^{\circ} \mathrm{C}$ in a $5 \% \mathrm{CO}_{2}$ humidified atmosphere. In the present study, the cells that had not migrated through the pores were manually removed from the upper face of the filters using cotton swabs. The cells that had invaded through the membrane were fixed in $90 \%$ alcohol and stained with $0.1 \%$ crystal violet for $5 \mathrm{~min}$, and then photographed under a microscope at a magnification of x200 (both from Olympus). The number of invaded cells was counted in five randomly selected fields.

Luciferase reporter assay. In brief, the miR-137-binding site in the CXCL12 3'-UTR region (wild- or mutant-type) was cloned and inserted into downstream of the firefly luciferase gene in a pGL3-promoter vector (Ambion, Austin, TX, USA). For the luciferase assays, $1 \times 10^{5} \mathrm{~K} 1$ cells were seeded into 24 -well plates and cultured for $24 \mathrm{~h}$. Then, the cells were co-transfected with $100 \mathrm{ng}$ of wild-type/mutant-type reporter plasmid, and $100 \mathrm{nM}$ of miR-137/miR-NC using Lipofectamine 2000 according to the manufacturer's protocol. At $48 \mathrm{~h}$ after transfection, Renilla luciferase activities in the cell lysates were measured using the Dual Luciferase Reporter Assay System (Promega, Madison, WI, USA).

Western blotting. The transfected cells were harvested, washed and lysed with RIPA buffer (Beyotime, Shanghai, China). Protein concentrations were measured using the bicinchoninic acid protein assay kit (Beyotime). Equivalent quantities $(30 \mu \mathrm{g})$ of protein were separated by $10 \%$ sodium dodecyl sulfate-polyacrylamide gel electrophoresis (SDS-PAGE) and transferred onto nitrocellulose membranes (Santa Cruz Biotechnology, Inc., Santa Cruz, CA, USA). The membranes were blocked with $5 \%$ non-fat milk in Tris-buffered saline for $2 \mathrm{~h}$ and incubated overnight at $4^{\circ} \mathrm{C}$ with primary antibodies against CXCL12 $(1: 1,000)$ and GAPDH $(1: 5,000)$ (both 


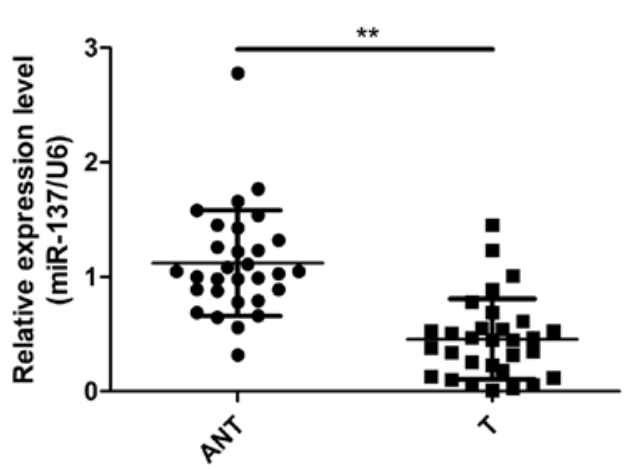

Figure 1. Expression of miR-137 is downregulated in human papillary thyroid carcinoma (PTC) tissues. miR-137 expression in 30 paired primary PTC tissues (T) and the matched adjacent normal tissues (ANT) from the same patients as determined by quantitative RT-PCR (qRT-PCR) analysis. ${ }^{* *} \mathrm{P}<0.01$ vs. ANT.

from Santa Cruz). The membranes were washed 3 times in TBS-Tween-20 and incubated with the corresponding horseradish peroxidase (HRP)-conjugated secondary antibody at a 1:5,000 dilution for $1 \mathrm{~h}$. The membranes were washed and probed with the secondary antibody conjugated to horseradish peroxidase (Santa Cruz) at a 1:5,000 dilution for $2 \mathrm{~h}$ at room temperature. Proteins were visualized using chemiluminescence detection (SignaGen, Rockville, MD, USA).

Statistical analysis. The data are expressed as the mean \pm SD (standard deviation) of 3 independent experiments. Group differences were compared using two-tailed Student's t-test or one-way ANOVA from SPSS version 19.0 software (SPSS, Inc., Chicago, IL, USA). A P-value of $<0.05$ was considered to indicate a statistically significant result

\section{Results}

miR-137 is downregulated in PTC tissues. To determine whether miR-137 is involved in PTC tumorigenesis, we detected the expression levels of miR-137 in the PTC and corresponding adjacent normal tissues. qRT-PCR assay showed that expression of miR-137 was significantly decreased in the PTC tissues compared with that in the adjacent normal tissues (Fig. 1). To further investigate the clinical relevance of miR-137 in PTC, the median value (0.46) of all 30 PTC samples was chosen as the cut-off point for grouping PTC patients with low miR-137 expression $(<0.46,16$ cases) and high miR-137 expression ( $>0.46,14$ cases). Then, by the Chi-square test, correlations between miR-137 expression and clinicopathological parameters were analyzed. It was found that miR-137 expression was significantly negatively associated with tumor-node-metastasis $(\mathrm{TNM})$ stage $(\mathrm{P}<0.01)$ and lymph node metastasis $(\mathrm{P}<0.01)$, which are both indicators of poor prognosis (Table I). However, no significant correlations were found between miR-137 expression and patient age and gender, as well as tumor size.

miR-137 inhibits cell proliferation and colony formation in the PTC cells. To investigate the biological function of miR-137 in PTC, we restored its expression by transfection of the miR-137 mimic in the K1 cells (Fig. 2A). Then, cell
Table I. Correlation between clinicopathological features and miR-137 expression in the PTC cases.

\begin{tabular}{|c|c|c|c|c|}
\hline \multirow[b]{2}{*}{ Variables } & \multirow[b]{2}{*}{$\begin{array}{c}\text { No. of } \\
\text { cases }\end{array}$} & \multicolumn{2}{|c|}{ miR-137 expression } & \multirow[b]{2}{*}{$\mathrm{P}$-value } \\
\hline & & $\begin{array}{l}\text { Low } \\
\mathrm{n}(\%)\end{array}$ & $\begin{array}{l}\text { High } \\
\text { n (\%) }\end{array}$ & \\
\hline Age (years) & & & & $>0.05$ \\
\hline$<55$ & 10 & $6(60.0)$ & $4(40.0)$ & \\
\hline$\geq 55$ & 20 & $10(50.0)$ & $10(50.0)$ & \\
\hline Gender & & & & $>0.05$ \\
\hline Male & 12 & $6(50.0)$ & $6(50.0)$ & \\
\hline Female & 18 & $10(55.6)$ & $8(44.4)$ & \\
\hline TNM stage & & & & $<0.01$ \\
\hline $\mathrm{T} 1-\mathrm{T} 2$ & & $8(38.1)$ & $13(62.9)$ & \\
\hline T3-T4 & & $8(88.9)$ & $1(11.1)$ & \\
\hline Tums & & & & $>0.05$ \\
\hline$<3$ & & $7(43.8)$ & $9(56.2)$ & \\
\hline$\geq 3$ & & $9(64.3)$ & $5(35.7)$ & \\
\hline I ymn & & & & $<0.01$ \\
\hline No & 22 & $8(36.4)$ & $14(63.6)$ & \\
\hline Yes & 8 & $8(100)$ & $0(0)$ & \\
\hline
\end{tabular}

PTC, papillary thyroid carcinoma.

proliferation and colony formation were determined in the $\mathrm{K} 1$ cells after transfection with miR-137 or miR-NC. It was found that restoration of miR-137 in K1 cells significantly reduced cell proliferation (Fig. 2B) and colony formation (Fig. 2C)

miR-137 inhibits cell migration and invasion in the PTC cells. To investigate whether miR-137 has an effect on migration and invasion in K1 cells, wound heal and Transwell chamber assays, respectively, were performed in $\mathrm{K} 1$ cells after transfection with miR-137 or miR-NC. It was found that overexpression of miR-137 in the K1 cells significantly suppressed cell migratory (Fig. 3A) and invasive (Fig. 3B) capabilities.

CXCL12 is a direct target of $m i R-137$. We investigated the candidate targets for miR-137 using prediction algorithms (Targetscan6.2 and miRanda). We selected CXCL12 for further validation since CXCL12 mRNA has one potential complimentary miR-137 binding site within its 3'-UTR region (Fig. 4A). We then performed a luciferase-based assay to validate whether this gene was regulated by miR-137. It was found that restoration of miR-137 expression in K1 cells obviously suppressed the luciferase activity of the wild-type CXCL12 site, while activity of the mutant CXCL12 site was not affected (Fig. 4B), which suggested that CXCL12 is directly targeted by miR-137. To determine whether miR-137 regulates CXCL12 expression in PTC cells, CXCL12 expression at the mRNA and protein levels was determined in $\mathrm{K} 1$ cells transfected with miR-137 or miR-NC by qRT-PCR and western blotting, respectively. As expected, restoration of 

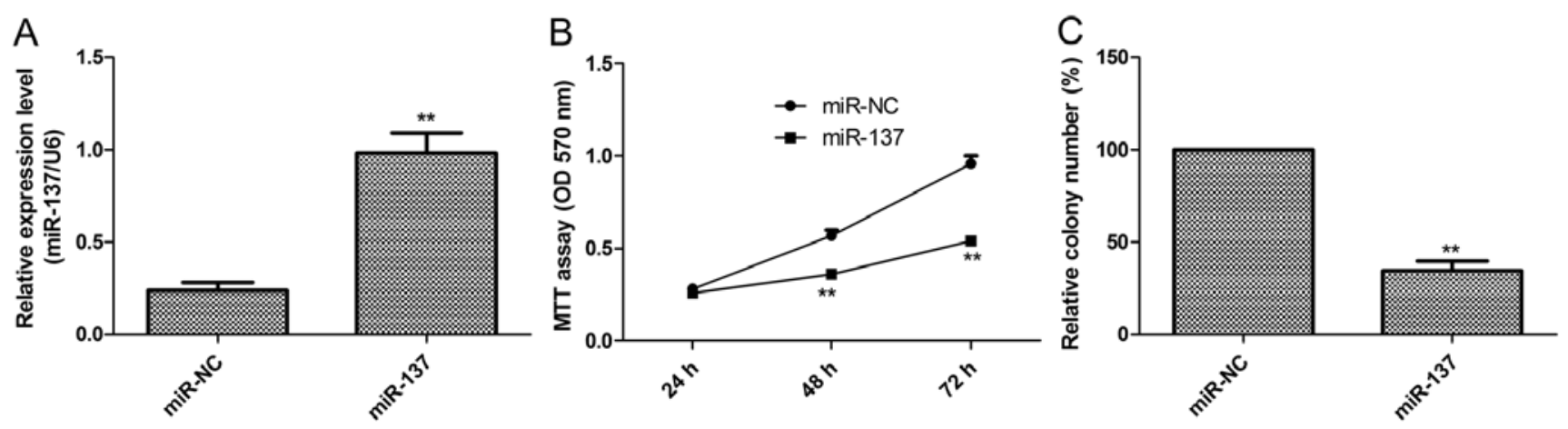

Figure 2. Restoration of miR-137 inhibits cell proliferation and colony formation in papillary thyroid carcinoma (PTC) cells. (A) miR-137 expression levels were determined in K1 cells after transfection with miR-137 or miR-NC by qRT-PCR analysis. (B) Cell proliferation was determined in K1 cells after transfection with miR-137 or miR-NC by MTT assay. (C) Cell colony formation was determined in K1 cells after transfection with miR-137 or miR-NC. ${ }^{* *} \mathrm{P}<0.01$ vs. miR-NC.

A

miR-NC

$\operatorname{miR}-137$

$\mathrm{Oh}$
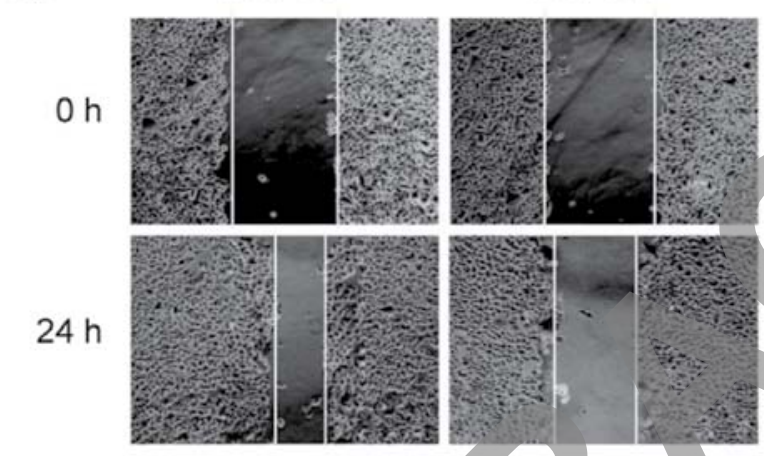

B
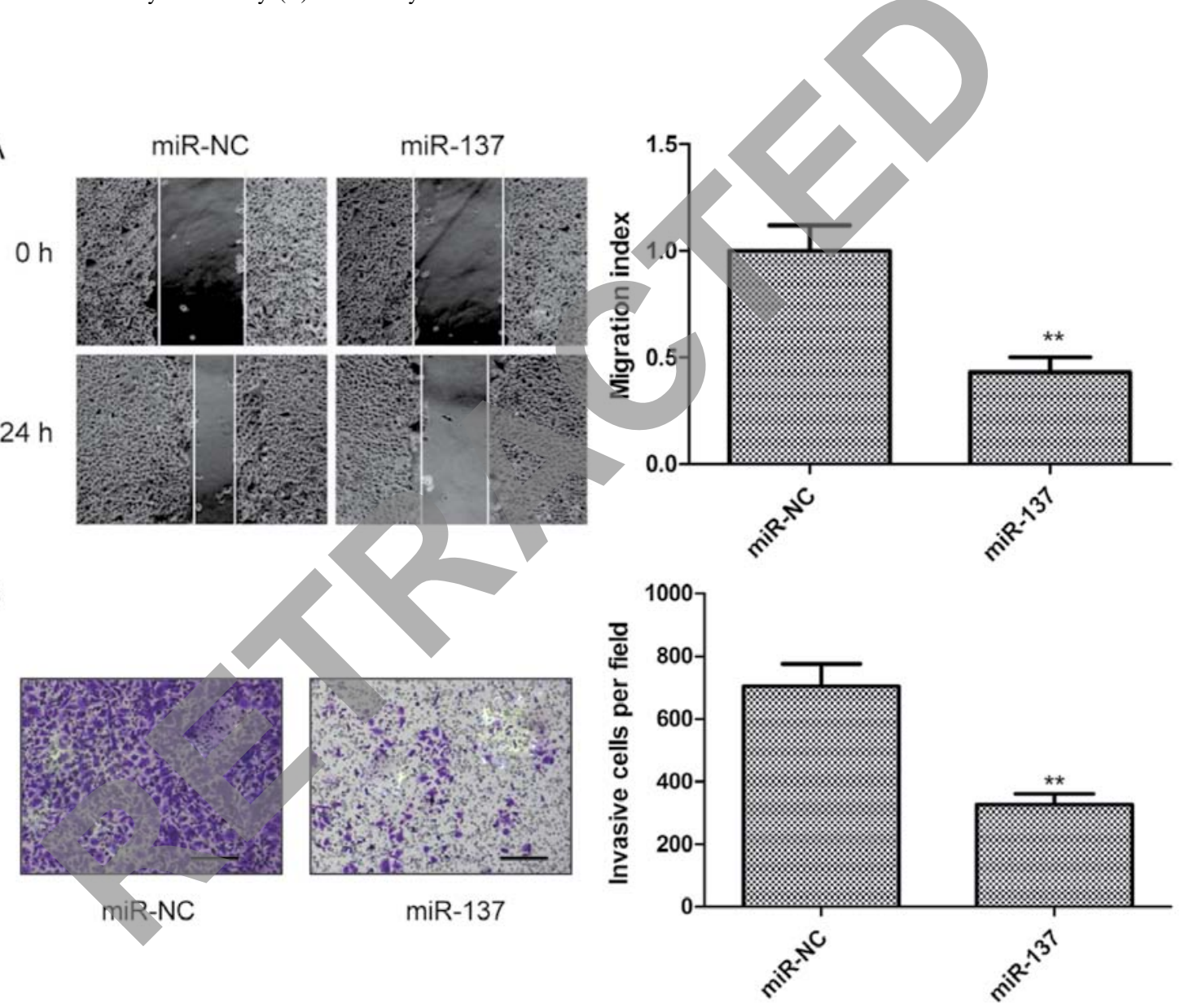

Figure 3. Restoration of miR-137 inhibits papillary thyroid carcinoma (PTC) cell migration and invasion. (A) Cell migration was determined in K1 cells after transfection with miR-137 or miR-NC by wound healing assay. (B) Cell invasion was determined in K1 cells after transfection with miR-137 or miR-NC by invasion chamber assay. ${ }^{* *} \mathrm{P}<0.01$ vs. miR-NC. Scale, $50 \mu \mathrm{m}$.

miR-137 in K1 cells obviously inhibited CXCL12 expression at the mRNA level (Fig. 4C) and protein level (Fig. 4D). These results indicated that CXCL12 is a direct target of miR-137 in PTC cells.

CXCL12 expression is upregulated and is inversely correlated with miR-137 expression in the PTC tissues. The above results suggested that CXCL12 is a direct target of miR-137 in PTC cells. Thus, we investigated the expression of CXCL12 in PTC and corresponding normal tissues by qRT-PCR and western blotting. CXCL12 expression was upregulated at the mRNA level (Fig. 5A) and protein level (Fig. 5B) in the PTC tissues compared with levels in the adjacent normal tissues. In addition, the miR-137 mRNA expression level was inversely correlated with miR-137 expression in the PTC tissues by Spearman's correlation analysis (Fig. 5C; $r=-0.549 ; \mathrm{P}<0.01$ ).

Knockdown of CXCL12 exhibits an effect similar to miR-137 overexpression in PTC cells. To explore the biological functions of CXCL12 in PTC cells, endogenous 


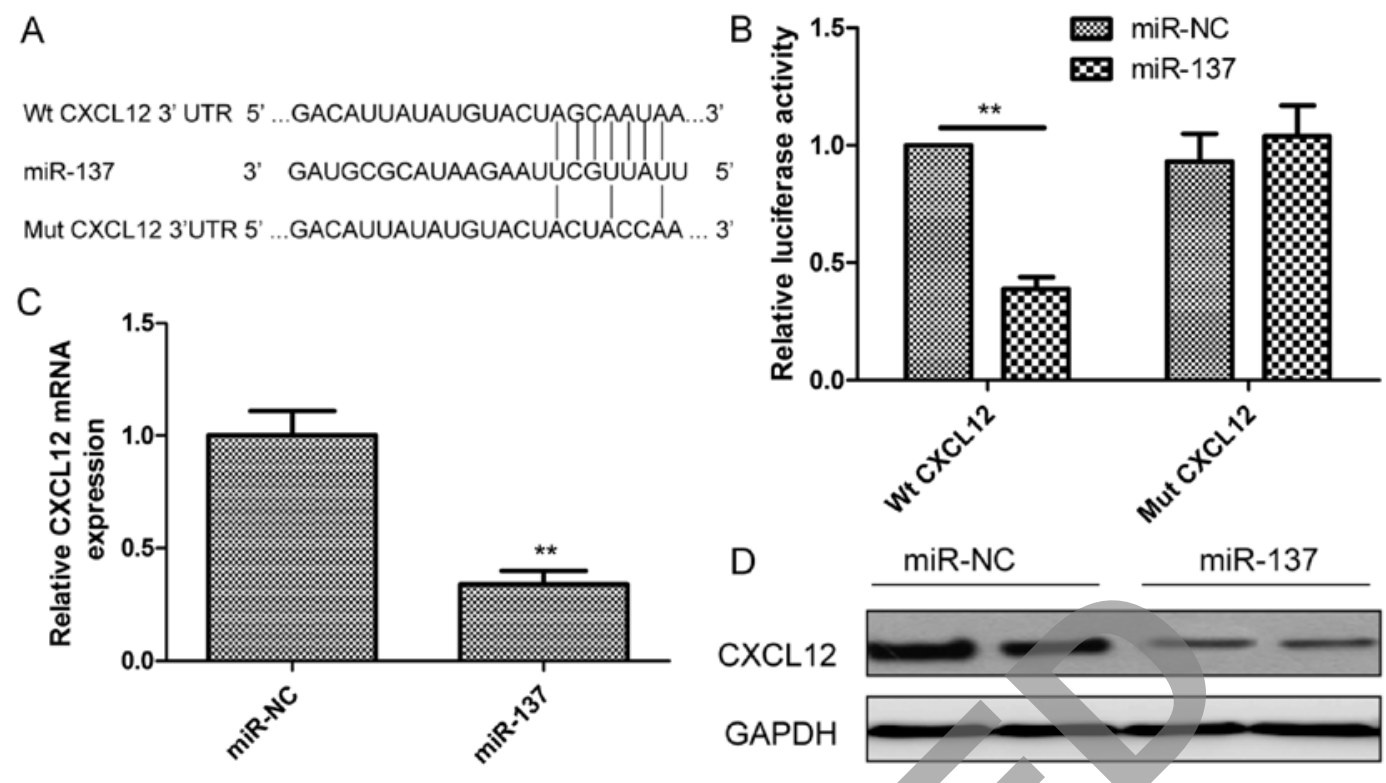

Figure 4. miR-137 suppresses CXCL12 expression by directly targeting the CXCL12 3' UTR, (A) Predicted miR-137 target sequence in the 3' UTR of CXCL12 and mutations in the binding sites are shown. (B) Luciferase reporter assay of the K1 cells co-transfected with the wild-type/mutant-type CXCL12 reporter plasmid and miR-137/miR-NC. Wt, wild-type; Mut, mutant-type. (C) qRT-PCR analysis of expression of CXCL12 in K1 cells transfected with miR-137 or miR-NC. GAPDH served as the loading control. (D) Western blot analysis of CXCL12 expression in K1 cells transfected with miR-137 or miR-NC. GAPDH served as the loading control. ${ }^{* *} \mathrm{P}<0.01$ vs. miR-NC.
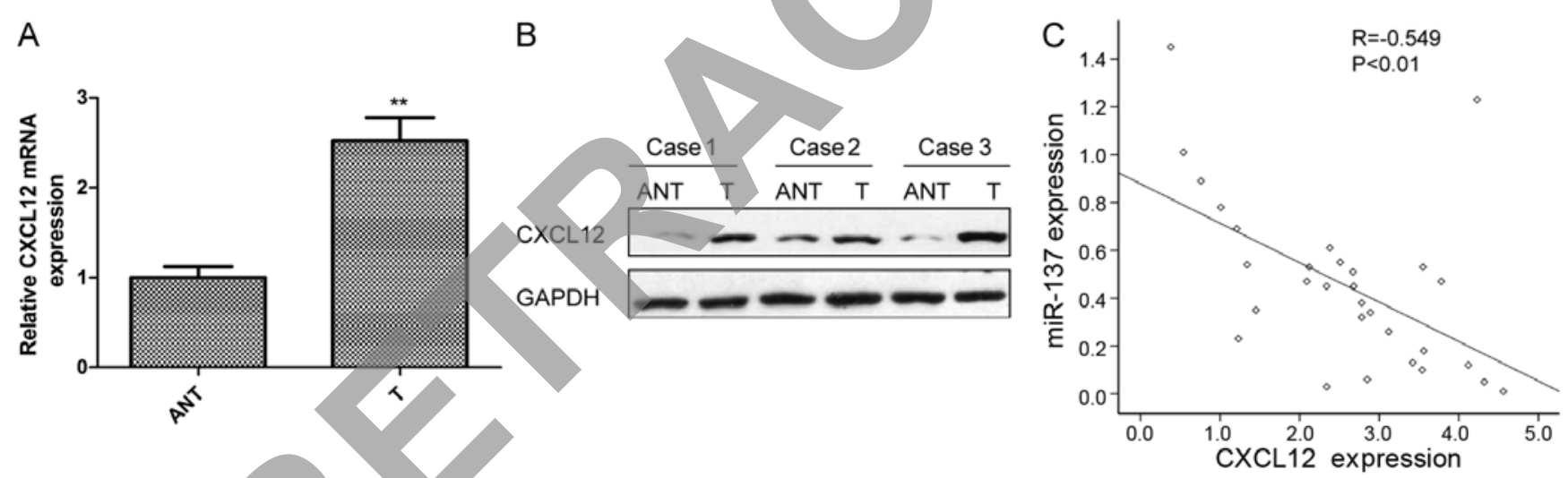

Figure 5. CXCL12 mRNA expression is upregulated and is inversely correlated with miR-137 expression in PTC tissues. (A) qRT-PCR analysis of mRNA expression of CXCL12 in 30 paired primary PTC tissues (T) and the matched adjacent normal tissues (ANT) from the same patient. GAPDH was used as an internal control. ${ }^{* *} \mathrm{P}<0.01$ vs. ANT. (B) Western blot analysis of CXCL12 protein expression in primary PTC tissues ( $\mathrm{T}$ ) and the matched adjacent normal tissues (ANT) from the same patient. (C) The inverse relationship between CXCL12 mRNA expression and miR-137 expression was explored in PTC tissues by Spearman's correlation.

expression of CXCL12 was knocked down in K1 cells with specific siRNA against CXCL12 (si-CXCL12), and then cell proliferation, colony formation, migration and invasion were assessed. Knockdown of CXCL12 significantly inhibited cell proliferation (Fig. 6A), colony formation (Fig. 6B), as well as decreased cell migration (Fig. 6C) and invasion (Fig. 6D) capabilities, suggesting that inhibition of CXCL12 mimicked the inhibitory effect of miR-137 overexpression in the K1 cells.

CXCL12 overexpression attenuates the suppressive effect of miR-137 in PTC cells. To evaluate whether the negative role of miR-137 on PTC was due to its regulation of CXCL12, we overexpressed miR-137, which subsequently strengthened CXCL12 expression in K1 cells. Our results showed that forced CXCL12 expression partially abrogated the inhibitory effects of miR-137 on cell proliferation, colony formation, migration and invasion (Fig. 7A-D). These results indicated that miR-137 exerts suppressive effects on PTC cells partially by targeting CXCL12.

\section{Discussion}

Accumulating evidence indicates that the aberrant expression of miRNAs contributes to papillary thyroid carcinoma (PTC) tumorigenesis and metastasis through repression of their target genes, suggesting that miRNAs may serve as molecular biomarkers for the prediction and prognosis of PTC, and as novel targets for disease treatment (12). For example, overexpression of miR-199a-3p in PTC cells was found to reduce MET and mTOR protein levels, impair migration and 

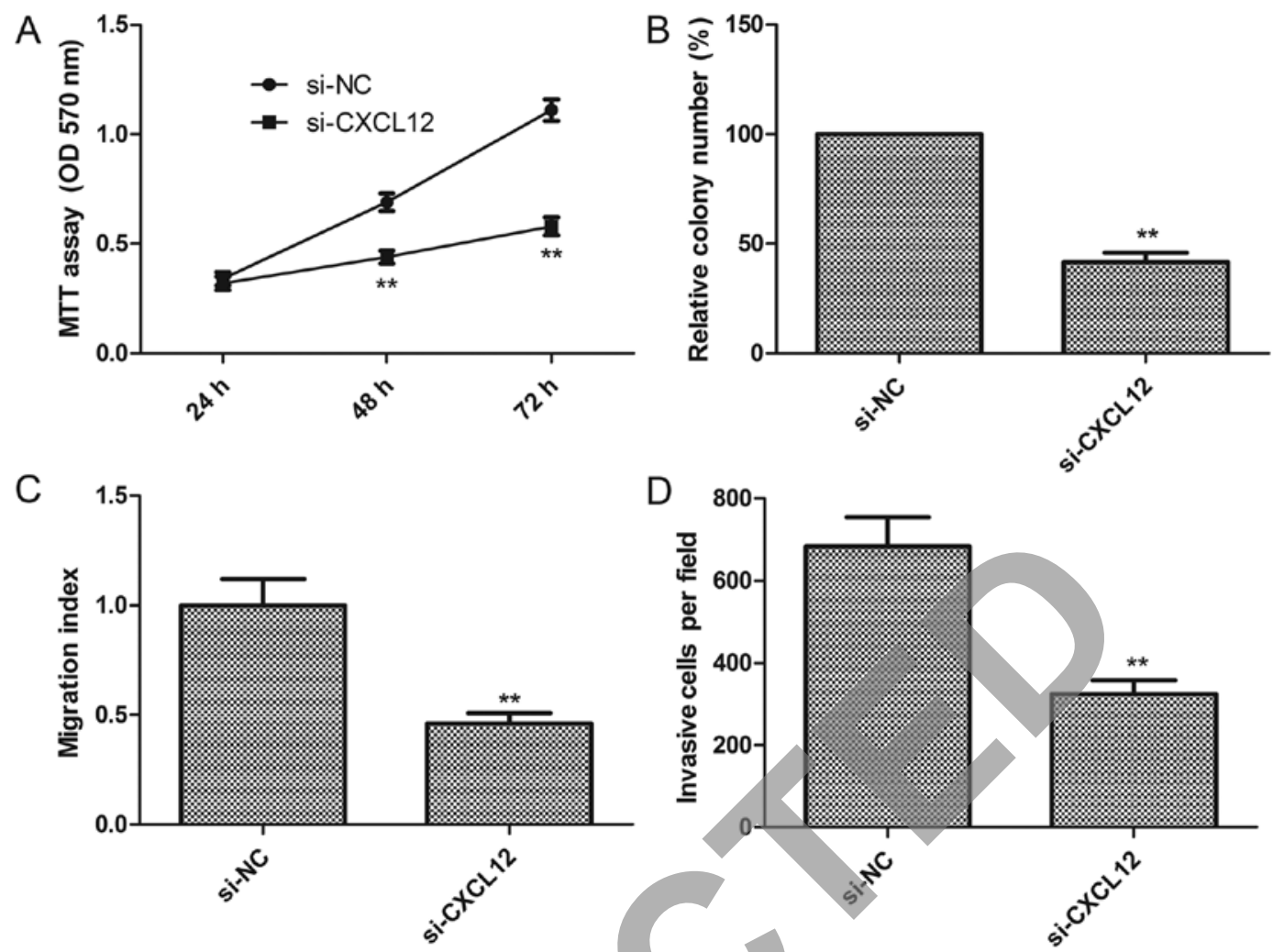

Figure 6. Downregulation of CXCL12 mimics the effect of miR-217 overexpression in papillary thyroid carcinoma (PTC) cells. Cell proliferation (A), colony formation (B), migration (C) and invasion (D) were determined in K1 cells transfected with si-CXCL12 or si-NC. ${ }^{* *} \mathrm{P}<0.01$ compared to si-NC.
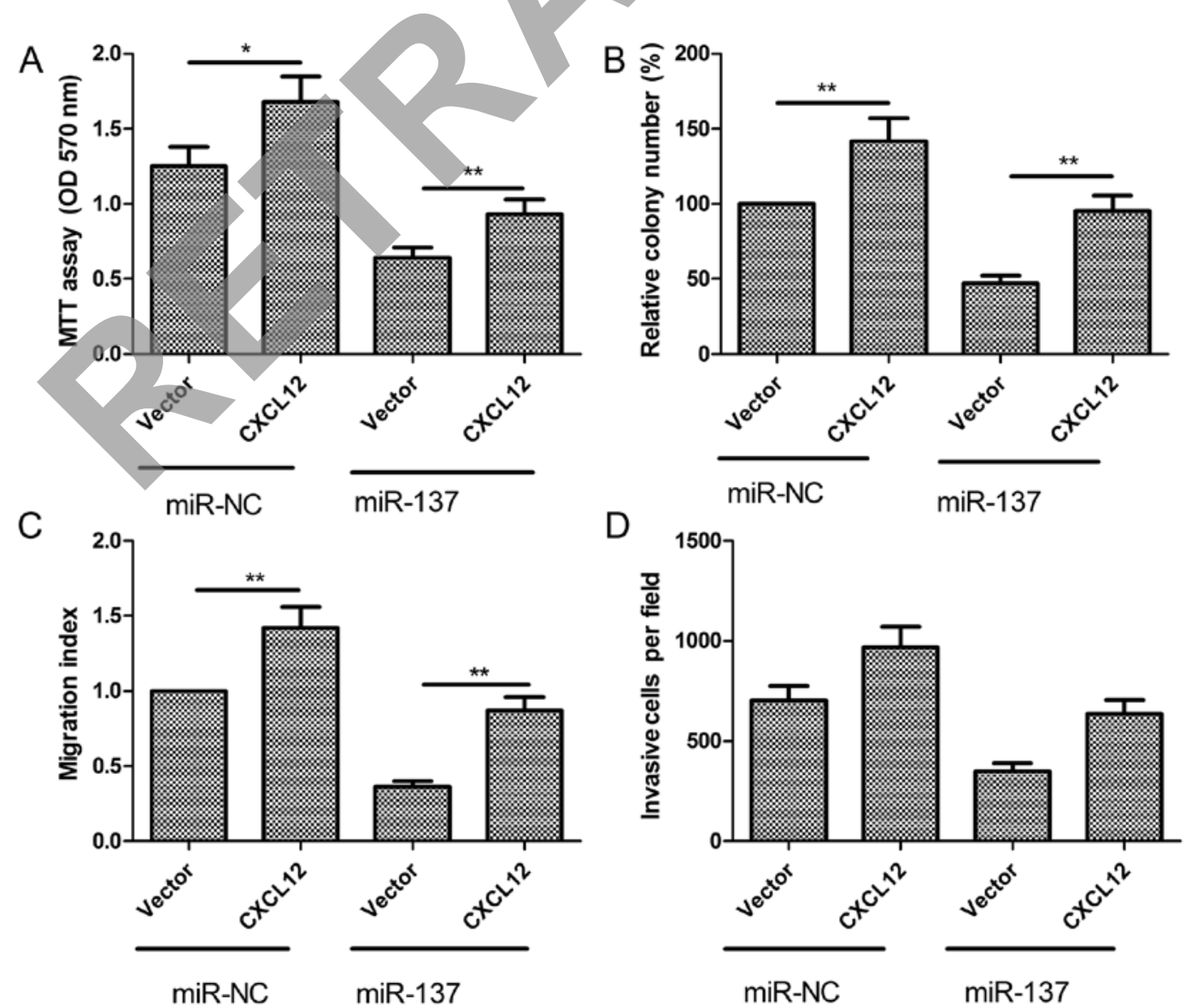

Figure 7. Overexpression of CXCL12 rescues the suppressive effects of miR-137 in papillary thyroid carcinoma K1 cells. K1 cells were transfected with miR-NC or the miR-137 mimic for $24 \mathrm{~h}$ followed by transfection with the empty vector and overexpression CXCL2 plasmid. Cell proliferation (A), colony formation (B), migration (C) and invasion (D) were determined in the cells. ${ }^{*} \mathrm{P}<0.05,{ }^{* *} \mathrm{P}<0.01$ compared to miR-137. 
proliferation and induce lethality through an unusual form of cell death similar to methuosis, caused by macropinocytosis dysregulation (21). Upregulation of miR-146b significantly promoted cell migration and invasiveness and increased resistance to chemotherapy-induced apoptosis in PTC (22). Restoration of miR-204-5p expression inhibited cell viability and colony formation efficiency, blocked cell cycle progression and enhanced apoptosis in vitro and suppressed tumorigenicity in vivo (23). miR-34a regulated growth arrest-specific 1 (GAS1) expression to promote proliferation and suppress apoptosis in PTC cells via the PI3K/Akt/Bad pathway (24). In the present study, we demonstrated that miR-137 was significantly downregulated in human PTC tissues, and its expression was significantly negatively correlated with TNM stage and lymph node metastasis. We also found that restoration of expression of miR-137 markedly suppressed the malignancy of PTC through inhibition of cell proliferation, colony formation, migration and invasion. These results suggest that miR-137 is a potential therapeutic target for PTC.

Recently, a number of studies have shown that the roles of miR-137 may be controversial in different types of cancers. miR-137 expression was reported to be upregulated in bladder cancer (25), and it was considered to be an oncomiR. However, in the majority of cancers, such as gastric (13), colorectal (14), non-small cell lung (15) and ovarian cancer (16), neuroblastoma (17), breast cancer (18) and hepatocellular carcinoma (26); miR-137 was suggested to function as a tumor suppressor. These studies suggest that miR-137 may play different roles depending on specific tumor type. However, the detailed biological function and underlying molecular mechanisms of miR-137 in PTC remain largely unclear. In the present study, we first found that miR-137 expression was downregulated in PTC tissues. In addition, we also found that restoration of miR-137 in PTC cells inhibited cell proliferation, colony formation, reduced cell migration and invasion capabilities by targeting CXCL12. These results suggest that miR-137 functions as a tumor suppressor in PTC.

CXCL12, also known as stromal-derived factor-1 (SDF-1), is a potent chemoattractant for hematopoietic cells and is important for cancer cell migration (27). It can bind to its receptor, CXCR4, leading to activation of the Src, PI3K/Akt, ERK and JNK pathways, contributing to protease production and cellular migration and invasion (28). It has been shown that CXCL12 and its receptors are involved in PTC progression and are markers for poor prognosis $(29,30)$. Importantly, CXCL12 has been identified as a direct target of several miRNAs, such as miR-23a (20), miR-518c-5p (31), miR-101 (32) and miR-448 (33). In the present study, we identified CXCL12 as a direct target of miR-137 by luciferase reporter assay. Restoration of miR-137 expression in K1 cells inhibited CXCL12 expression at the mRNA and protein levels. We also showed that CXCL12 expression was upregulated in PTC tissues, and its expression was inversely correlated with miR-137 expression. Notably, we found that inhibition of CXCL12 expression had a similar effect as that of restored miR-137 expression in K1 cells; and that reintroduction of CXCL12 partially abrogated the suppressive effect induced by miR-137 in K1 cells. These results suggest that miR-137 exerts suppressive effects in human PTC partially through suppression of CXCL12.
In summary, the present study identified miR-137 as a tumor suppressive miRNA in human PTC at least partly through the targeting of CXCL12. These findings may provide a new therapeutic strategy for human PTC.

\section{References}

1. Brown RL, de Souza JA and Cohen EE: Thyroid cancer: Burden of illness and management of disease. J Cancer 2: 193-199, 2011.

2. Pitoia F, Bueno F, Urciuoli C, Abelleira E, Cross G and Tuttle RM: Outcomes of patients with differentiated thyroid cancer risk-stratified according to the American Thyroid Association and Latin American Thyroid Society Risk of Recurrence Classification Systems. Thyroid 23: 1401-1407, 2013.

3. Nikiforova MN and Nikiforov YE: Molecular genetics of thyroid cancer: Implications for diagnosis, treatment and prognosis. Expert Rev Mol Diagn 8: 83-95, 2008.

4. Lang BH, Wong KP, Wan KY and Lo CY: Significance of metastatic lymph node ratio on stimulated thyroglobulin levels in papillary thyroid carcinoma after prophylactic unilateral central neck dissection. Ann Surg Oncol 19: 1257-1263, 2012.

5. Brennecke $\mathrm{J}$ and Cohen SM: Towards a complete description of the microRNA complement of animal genomes. Genome Biol 4: $228,2003$.

6. Ambros V: The functions of animal microRNAs. Nature 431: 350-355, 2004.

7. Bartel DP: MicroRNAs: Genomics, biogenesis, mechanism, and function. Cell 116: 281-297, 2004.

8. Carthew RW and Sontheimer EJ: Origins and mechanisms of miRNAs and siRNAs. Cell 136: 642-655, 2009.

9. Esquela-Kerscher A and Slack FJ: Oncomirs - microRNAs with a role in cancer. Nat Rev Cancer 6: 259-269, 2006.

0. Lu J, Getz G, Miska EA, Alvarez-Saavedra E, Lamb J, Peck D, Sweet-Cordero A, Ebert BL, Mak RH, Ferrando AA, et al: MicroRNA expression profiles classify human cancers. Nature 435: 834-838, 2005

11. Volinia S, Calin GA, Liu CG, Ambs S, Cimmino A, Petrocca F, Visone R, Iorio M, Roldo C, Ferracin M, et al: A microRNA expression signature of human solid tumors defines cancer gene targets. Proc Natl Acad Sci USA 103: 2257-2261, 2006.

12. Aragon Han P, Weng $\mathrm{CH}$, Khawaja HT, Nagarajan $\mathrm{N}$, Schneider EB, Umbricht CB, Witwer KW and Zeiger MA: MicroRNA expression and association with clinicopathologic features in papillary thyroid cancer: A systematic review. Thyroid 25: 1322-1329, 2015.

13. Chen Q, Chen X, Zhang M, Fan Q, Luo S and Cao X: miR-137 is frequently down-regulated in gastric cancer and is a negative regulator of Cdc42. Dig Dis Sci 56: 2009-2016, 2011.

14. Liu M, Lang N, Qiu M, Xu F, Li Q, Tang Q, Chen J, Chen X, Zhang S, Liu Z, et al: miR-137 targets Cdc42 expression, induces cell cycle G1 arrest and inhibits invasion in colorectal cancer cells. Int J Cancer 128: 1269-1279, 2011.

15. Bi Y, Han Y, Bi H, Gao F and Wang X: miR-137 impairs the proliferative and migratory capacity of human non-small cell lung cancer cells by targeting paxillin. Hum Cell 27: 95-102, 2014.

16. Guo J, Xia B, Meng F and Lou G: miR-137 suppresses cell growth in ovarian cancer by targeting AEG-1. Biochem Biophys Res Commun 441: 357-363, 2013.

17. Althoff K, Beckers A, Odersky A, Mestdagh P, Köster J, Bray IM, Bryan K, Vandesompele J, Speleman F, Stallings RL, et al: MiR-137 functions as a tumor suppressor in neuroblastoma by downregulating KDM1A. Int J Cancer 133: 1064-1073, 2013.

18. Zhao Y, Li Y, Lou G, Zhao L, Xu Z, Zhang Y and He F: MiR-137 targets estrogen-related receptor alpha and impairs the proliferative and migratory capacity of breast cancer cells. PLoS One 7: e39102, 2012.

19. Zhang L, Li Z, Gai F and Wang Y: MicroRNA-137 suppresses tumor growth in epithelial ovarian cancer in vitro and in vivo. Mol Med Rep 12: 3107-3114, 2015.

20. He Y, Meng C, Shao Z, Wang $\mathrm{H}$ and Yang S: MiR-23a functions as a tumor suppressor in osteosarcoma. Cell Physiol Biochem 34: 1485-1496, 2014.

21. Minna E, Romeo P, De Cecco L, Dugo M, Cassinelli G, Pilotti S, Degl'Innocenti D, Lanzi C, Casalini P, Pierotti MA, et al: miR-199a-3p displays tumor suppressor functions in papillary thyroid carcinoma. Oncotarget 5: 2513-2528, 2014. 
22. Chou CK, Yang KD, Chou FF, Huang CC, Lan YW, Lee YF, Kang HY and Liu RT: Prognostic implications of miR-146b expression and its functional role in papillary thyroid carcinoma. J Clin Endocrinol Metab 98: E196-E205, 2013.

23. Liu L, Wang J, Li X, Ma J, Shi C, Zhu H, Xi Q, Zhang J, Zhao X and $\mathrm{Gu} \mathrm{M}$ : MiR-204-5p suppresses cell proliferation by inhibiting IGFBP5 in papillary thyroid carcinoma. Biochem Biophys Res Commun 457: 621-626, 2015.

24. Ma Y, Qin H and Cui Y: MiR-34a targets GAS1 to promote cell proliferation and inhibit apoptosis in papillary thyroid carcinoma via PI3K/Akt/Bad pathway. Biochem Biophys Res Commun 441: 958-963, 2013.

25. Xiu Y, Liu Z, Xia S, Jin C, Yin H, Zhao W and Wu Q: MicroRNA-137 upregulation increases bladder cancer cell proliferation and invasion by targeting PAQR3. PLoS One 9: e109734, 2014

26. Liu LL, Lu SX, Li M, Li LZ, Fu J, Hu W, Yang YZ, Luo RZ, Zhang CZ and Yun JP: FoxD3-regulated microRNA-137 suppresses tumour growth and metastasis in human hepatocellular carcinoma by targeting AKT2. Oncotarget 5: 5113-5124, 2014.

27. Secchiero P, Celeghini C, Cutroneo G, Di Baldassarre A, Rana R and Zauli G: Differential effects of stromal derived factor-1 alpha (SDF-1 alpha) on early and late stages of human megakaryocytic development. Anat Rec 260: 141-147, 2000.
28. Nagasawa T: CXCL12/SDF-1 and CXCR4. Front Immunol 6: 301,2015

29. Jung YY, Park IA, Kim MA, Min HS, Won JK and Ryu HS: Application of chemokine CXC motif ligand 12 as a novel diagnostic marker in preoperative fine-needle aspiration biopsy for papillary thyroid carcinoma. Acta Cytol 57: 447-454, 2013.

30. Mochizuki H, Matsubara A, Teishima J, Mutaguchi K, Yasumoto H, Dahiya R, Usui T and Kamiya K: Interaction of ligand-receptor system between stromal-cell-derived factor-1 and CXC chemokine receptor 4 in human prostate cancer: A possible predictor of metastasis. Biochem Biophys Res Commun 320: 656-663, 2004.

31. Kinouchi M, Uchida D, Kuribayashi N, Tamatani T, Nagai H and Miyamoto Y: Involvement of miR-518c-5p to growth and metastasis in oral cancer. PLoS One 9: e115936, 2014.

32. Zhang J, Liu J, Liu Y, Wu W, Li X, Wu Y, Chen H, Zhang K and Gu L: miR-101 represses lung cancer by inhibiting interaction of fibroblasts and cancer cells by down-regulating CXCL12. Biomed Pharmacother 74: 215-221, 2015.

33. Lv Y, Lei Y, Hu Y, Ding W, Zhang C and Fang C: miR-448 negatively regulates oyarian cancer cell growth and metastasis by targeting CXCL12. Clin Transl Oncol 17: 903-909, 2015. 